\title{
Synaptic Microenvironment in Depressive Disorder: Insights from Synaptic Plasticity
}

This article was published in the following Dove Press journal:

Neuropsychiatric Disease and Treatment

\author{
Feifei Ren (D) \\ Rongjuan Guo ${ }^{2}$ \\ 'Second Clinical Medical College, Beijing \\ University of Chinese Medicine, Beijing, \\ I00029, People's Republic of China; \\ ${ }^{2}$ Department of Neurology, Dongfang \\ Hospital, Beijing University of Chinese \\ Medicine, Beijing 100078, People's \\ Republic of China
}

\begin{abstract}
Depression is a major disease that can affect both mental and physical health, limits psychosocial functioning and diminishes the quality of life. But its complex pathogenesis remains poorly understood. The dynamic changes of synaptic structure and function, known as synaptic plasticity, occur with the changes of different cellular microenvironment and are closely related to learning and memory function. Accumulating evidence implies that synaptic plasticity is integrally involved in the pathological changes of mood disorders, especially in depressive disorder. However, the complex dynamic process of synaptic plasticity is influenced by many factors. Here, we reviewed and discussed various factors affecting synaptic plasticity in depression, and proposed a specific framework named synaptic microenvironment, which may be critical for synaptic plasticity under pathological conditions. Based on this concept, we will show how we understand the balance between the synaptic microenvironment and the synaptic plasticity network in depression. Finally, we point out the clinical significance of the synaptic microenvironment in depression.
\end{abstract}

Keywords: depression, synaptic plasticity, synaptic microenvironment, glia, influence factors

\section{Introduction}

Depression is nowadays a major common mental illness in most societies worldwide and has become an important public health problem. ${ }^{1}$ It will seriously affect physical and mental health. ${ }^{2}$ The World Health Organization estimates that more than 350 million people worldwide suffer from depression. ${ }^{3}$ According to the Global Burden of Disease Study 2017, depression was one of the leading level 3 causes of years lived with disability (YLDs) counts in 2017, whereas this was not the case in $1990 .{ }^{4}$ China has 56.36 million people suffering from depression in 2017 , accounting for $21.3 \%$ of total subjects in the world. ${ }^{5}$ However, many patients with depression do not receive extensive diagnosis and treatment, which is possibly due to ambiguous and confusing concepts, stigma, multimorbidity, lack of effective therapies, inadequate mental-health resources and the absence of reliable and valid biomarkers. ${ }^{6}$

Synaptic plasticity refers to the capacity of pre-existing connections between two neurons to change their original strength with neural activity. ${ }^{7}$ It has long been believed that synaptic plasticity in the adult brain represents the cellular mechanism of learning and memory. ${ }^{8}$ However, with the growing knowledge of mental illness, synaptic plasticity has been gradually found to be one of the important neural mechanisms of depression. ${ }^{9}$ Interestingly, mounting evidence suggests that synaptic
Correspondence: Rongjuan Guo Department of Neurology, Dongfang Hospital, Beijing University of Chinese Medicine, No. 6, Ist Section,

Fangxingyuan, Fangzhuang, Fengtai District, Beijing City, People's Republic of China

Tel/Fax +86 10-6768-9655

Email38II60563@qq.com
Neuropsychiatric Disease and Treatment 2021:17 |57-165 in $\bullet$ 
plasticity is often influenced by the synaptic microenvironment, such as astrocytes and microglia. ${ }^{10,11}$ Meanwhile, cytokines and their receptors can exert a regulatory effect of brain synaptic plasticity. ${ }^{12}$ In addition, synaptic remodeling depends on the signaling between the serotonin receptor and extracellular matrix, which is deeply associated with synaptic plasticity. ${ }^{13}$ In this review, we briefly evaluate the current studies on the role of synaptic plasticity in depression. More specifically, we discuss the role of immune signaling, oxidative stress, mitochondria, glia and neurotransmitter in synaptogenesis, and how these factors regulate synaptic plasticity as part of the synaptic microenvironment. Finally, we point out the clinical significance of the synaptic microenvironment in depression.

\section{Role of Synaptic Plasticity in Depression}

Synapses are the sites of contact and point-to-point information transmission between neurons and their partner cell. ${ }^{14}$ Each synapse is mainly composed of presynaptic structure, synaptic gap, postsynaptic structure and supporting cells, which act as a specialized neural junction. Presynaptic cells assemble the secretion mechanism of the neurotransmitter, while postsynaptic cells assemble components to receive and integrate this signal. ${ }^{15}$ During this dynamic process, the function and/or structure of synapses change, which is called synaptic plasticity and is also a key link in several neurological diseases.

Although considerable progress has been made in the pathophysiology of depression, there is no single mechanism that can explain all aspects of the disease satisfactorily so far. ${ }^{16}$ At present, it is believed that the decline of monoamine hypothesis transmitters system, enhancement of the hypothalamic-pituitary-adrenal (HPA) axis, inflammatory activation, decreased neuroplasticity and neurogenesis, changes in brain structure and function, genes, environmental milieu and epigenetics are involved in the pathogenesis of depression. ${ }^{17}$ In addition, synaptic plasticity may be another important aspect.

Existing studies have also shown that the occurrence of depression is closely related to changes in synaptic plasticity. ${ }^{18}$ This possibility has been found in preclinical and clinical studies of stress, depression and antidepressants.

Firstly, as we all know, chronic stress has a crucial role in the development of psychiatric diseases, such as anxiety and depression, and stress differentially affects the DSM-5 depressive symptoms. ${ }^{19,20}$ Even though none of the animal models developed so far perfectly reproduce the depression-like phenotype observed in humans, breakthroughs have still been found in rodents over the last few decades. ${ }^{21}$ Chronic stress can lead to a wide range of behavioral deficits in rodents, including decreased sucrose preference, motivation for reward stimuli and sexual behaviors, along with increased aggression, anxiety-like behaviors and altered sleep patterns. ${ }^{22}$ A preclinical study has shown that brain-derived neurotrophic factor (BDNF), which plays a key role in synaptic plasticity, is significantly reduced in the hippocampus tissue in chronic unpredictable stress (CUS) depressed model rats. ${ }^{23}$ Meanwhile, a study focused on the effects of authentic stress and transmitted stress on nerve synapses found that both of them in mice primed paraventricular nucleus of the hypothalamus (PVN) corticotropin-releasing hormone $(\mathrm{CRH})$ neurons, enabling the induction of metaplasticity at glutamate synapses. ${ }^{24}$ Another basic study shows that exposure to stress alters spine morphology, function and plasticity and that antidepressants, particularly new rapidacting agents, can reverse these effects. ${ }^{25}$ Also, early life stress (ELS) has been proved to impair fear memory in 6-month-old mice and decreased hippocampal long-term potentiation (LTP) synaptic plasticity as well as the pairedpulse ratio (PPR), and this mechanism is related to the reduction of hippocampal GluN2B expression. ${ }^{26}$

Secondly, clinical studies demonstrate that the volume of hippocampus and medial prefrontal cortex in depression patients is significantly reduced, neurons and glial cells atrophy with loss in vulnerable brain regions, such as hippocampus and prefrontal cortex, and the number of synapses is significantly lower. ${ }^{27} \mathrm{~A}$ prospective trial of 220 patients with major depressive disorder (MDD) who were non-responders to a previous antidepressant showed replicated expression of genes related to synaptic plasticity such as ZNF804A rs7603001, CREB1 rs2254137 and CHL1 rs2133402. ${ }^{28}$ A study using anatomical brain magnetic resonance imaging in a randomized, double-blind, placebo-controlled trial (RCT) of antidepressant medication for 10 weeks' duration in patients with dysthymia found that, at baseline, patients had diffusely thicker cortices than did healthy participants, and patients who had thicker cortices had proportionately less severe symptoms; these findings provide in vivo evidence within an RCT for the presence of neuroanatomical plasticity in depression. ${ }^{29}$

In addition, most antidepressants can significantly affect the levels of monoamine neurotransmitters secreted by synapses predominantly, and modulate synaptic 
transmission using the neurotransmitter glutamate, ${ }^{30}$ showing that antidepressants can regulate synaptic plasticity. As a rising star in the field of antidepressants, ketamine can induce rapid and sustained antidepressant action by regulating downstream mechanisms of synaptic plasticity, including BDNF, eukaryotic elongation factor 2 (eEF2), mechanistic target of rapamycin (mTOR) and glycogen synthase kinase-3 (GSK-3). ${ }^{31}$ Ketamine may act in a complementary manner to exert acute changes in synaptic plasticity in the CA3 subregion of the hippocampus, ${ }^{32}$ resulting in sustained strengthening of excitatory synapses, which are necessary for antidepressant behavior. In addition, a basic study has found that the prefrontal circuit plays a critical role in sustaining specific antidepressant behavioral effects and maintaining long-term behavioral remission through repeated longitudinal imaging of medial prefrontal microcircuits in the living brain, and antidepressant-dose ketamine reversed these effects by selectively rescuing eliminated spines and restoring coordinated activity in multicellular ensembles that predict motivated escape behavior. ${ }^{33}$ Also, a single injection of ketamine or its metabolite $(2 \mathrm{R}, 6 \mathrm{R})$-hydroxynorketamine $(2 \mathrm{R}, 6 \mathrm{R})$-HNK can induce enduring alterations in the function of AMPA receptors (AMPARs) and synaptic plasticity in brain regions involved in reward-related behaviors. ${ }^{34}$

\section{Gender Differences in Depression and Synaptic Plasticity}

Clinical studies have shown that women are more susceptible to depression than men. ${ }^{35}$ Gender differences in depression have been associated with social, cultural, as well as biological factors. ${ }^{36}$ One study indicated that neuroactive estrogenic and androgenic steroids influence synaptic transmission in both directions, finely modulating synaptic plasticity in several brain regions including the hippocampus CA1 region. While estrogens facilitate LTP, androgens are involved in the induction of longterm depression (LTD) and depotentiation (DP) of synaptic transmission. ${ }^{37}$ Another study using a 64channel multielectrode (MED64) system to record synaptic plasticity in the ACC of male and female adult mice found that LTD was greater in slices of ACC in male mice than in female mice. ${ }^{38}$ In addition, synaptic-plasticity protein markers, including postsynaptic density 95, synaptophysin and growth-associated binding protein 43, had significantly lower expression levels in the maternal separation (MS) group than in the non-MS (NMS) group. Metabolomics analysis shows that the MS model caused adult Sprague-Dawley (SD) rats to be susceptible to depression, which may regulate synaptic plasticity through arginine and proline metabolism; pantothenate and CoA biosyntheses; glutathione metabolism; and phenylalanine, tyrosine and tryptophan biosyntheses. All these studies reflect the impact of gender differences on synaptic plasticity in depression.

In spite of extensive preclinical studies in animal models for depression that have been used for understanding the mechanisms of the disease as well as for new drug development, a substantive lack of attention to sex-specific phenotypes in depression might mask the effect of gender on the outcome, even on the recurrence. ${ }^{39-41}$ So, a more systematic consideration of the influence for synaptic plasticity on biological gender as a variable in depression research will be essential in the discovery and development of pharmacotherapies that are effective for both men and women.

\section{Role of Synaptic Microenvironment in Synaptic Plasticity in Depression}

At a certain stage of neuron development, the differentiation of its chemical properties is plastic, and the formation of neurotransmitters and synapses will change accordingly. Of course, the differentiation of neurons and the changes of neuronal synaptic plasticity are not only affected by the transmission of information centers, but also closely related to the ion concentration of contact neurons and their surrounding cells, which we call the synaptic microenvironment.

The synaptic microenvironment refers to the neuron synapses and their surrounding environment at microscopic level. This concept usually needs to be understood from a holistic perspective, not only focusing on changes in the structure and function of synapses in diseases, but more importantly focusing on the impact of the surrounding environment on synapses, similar to the concept of the tumor microenvironment. Under this concept, changes in synaptic plasticity in depression not only focus on the remodeling of the synapse itself, but also focus on the influence of the environment around the synapse on the plasticity of the synapse. In such a microenvironment, the mechanism of interaction of many factors, and how to analyze it qualitatively and quantitatively, has always been an important proposition in neuropsychiatric research, and the same is true for depression research. 


\section{Structural Changes of Synaptic}

Microenvironment in Relation to Synaptic Plasticity

As the connection between neurons, synapses are closely related to their surrounding changes. We mainly focus on the extracellular matrix (ECM), neurovascular units and glial and other cells. These areas are briefly summarized and illustrated here.

Firstly, investigators have demonstrated that the ECM molecules, which are synthesized by neurons and glial and non-neuronal cells, form highly organized ECM structures around cell somata, axon initial segments and synapses and play a prominent role by regulating the closure of critical periods of synaptic plasticity. ${ }^{42,43}$ These molecules includetenascin R (TNR), reelin, chondroitin sulphate proteoglycans (CSPGs), tenascin-C (TN-C) and integrins. ${ }^{44-48}$ Also, the ECM plays a dual role, not only as an accelerator of structural and functional plasticity, but also as a biodegradable stabilizer of neural microcircuits, which is important for mental health. ${ }^{49}$ A study mechanistically links a signaling pathway involving the serotonin 5-HT7 receptor (5-HT7R), matrix metalloproteinase 9 (MMP-9), the hyaluronan receptor CD44 and the small GTPase Cdc42, and found that 5-HT7R stimulation increases local MMP-9 activity, triggering dendritic spine remodeling, synaptic pruning and impairment of LTP. ${ }^{50}$

Secondly, the neurovascular plasticity of hippocampus is an important theory underlying major depression. Published research shows that the neurovascular unit, which includes neurons, astrocytes, pericytes and microglia as well as the blood vessels themselves, participates actively in neurostructural changes and mediates the bioavailability of cytokines and molecular mediators, and is fundamental for the synaptic plasticity. ${ }^{51,52}$ A study investigating the structural plasticity of the hippocampus with ketamine found that neurovascular changes of the hippocampus could be one of the possible mechanisms underlying the sustained antidepressant effect of ketamine, by altering the number of the excitatory synapses as well as the neuronal number and length of the microvessels in the hippocampus. ${ }^{53}$ This fully confirms our argument.

Thirdly, synaptic plasticity is obviously influenced by three main non-neuronal cells: astrocytes, microglia and oligodendrocytes. Recent studies indicate that glial cells, which include astrocytes and microglia, tightly and dynamically interact with the processing of synaptic information in depression. ${ }^{54,55} \mathrm{~A}$ study identified that chordin-like
1 (Chrd11), which is restricted to cortical astrocytes in vivo, is necessary and sufficient to induce mature GluA2-containing synapses to form. Astrocytes, via the release of Chrdl1, promote GluA2-dependent synapse maturation and thereby limit synaptic plasticity. ${ }^{56}$ Another study expressed the Gq-coupled receptor hM3Dq in CA1 astrocytes, which can activate the astrocytes by a designer drug, and found that astrocytic activation is not only necessary for synaptic plasticity, but also sufficient to induce NMDA-dependent de novo LTP in the hippocampus that persisted after astrocytic activation ceased. ${ }^{57}$ Studies also confirmed the molecular interaction between neurons and microglia that drives experiencedependent synapse remodeling in the hippocampus. The cytokine interleukin-33 (IL-33), which is expressed by adult hippocampal neurons, defines a neuronal subset primed for synaptic plasticity. Also, the neuronal IL-33 instructs microglial engulfment of the extracellular matrix (ECM), and its loss leads to impaired ECM engulfment and a concomitant accumulation of ECM proteins in contact with synapses. ${ }^{58}$ Besides, NogoA is mainly expressed by oligodendrocytes, but also in the hippocampus where it is found at synaptic sites, which indicates that the oligodendrocytes also limited synaptic plasticity. ${ }^{59}$ A study using fusion proteins to track synaptic vesicle localization and membrane fusion in zebrafish during developmental myelination also investigated expression and localization of PSD95, a dendritic post-synaptic protein, within oligodendrocytes. ${ }^{60}$ This work raises the possibility that axon-glial communication contributes to myelin plasticity and is also part of synaptic plasticity. Studies have also shown that oligodendrocytes modulate neurotransmitter release at presynaptic terminals through secretion of BDNF. Oligodendrocyte-derived BDNF functions via presynaptic tropomyosin receptor kinase B (TrkB) to ensure fast, reliable neurotransmitter release and auditory transmission in the developing brain. ${ }^{61}$

In addition, the long-range interaction between immune cells and the central nervous system allows the immune system to engage the communication pathways of the nervous system, with immune cells and mediators playing a regulatory role in the nervous system, and also participating in the elimination and plasticity of synapses during development. ${ }^{62}$ When the activation of the peripheral immune system continues unabated, the ensuing immune signaling to the brain can lead to the development of depression symptoms, ${ }^{63}$ and the synapse acts as a bridge between immune cells and depression. 


\section{Functional Changes of Synaptic \\ Microenvironment in Relation to Synaptic Plasticity}

In addition to structural changes, variations of function are also an important aspect of the synaptic microenvironment, which affect synaptic plasticity in depression significantly, and, to some extent, functional diversity is also the result of structural modifications. Several proposed mechanisms are currently recognized as the causes of functional changes in the synaptic microenvironment in depression, such as abnormal immune signaling, excessive oxidative stress, mitochondrial dysfunction and cytokine changes.

Increasing amounts of data suggest that abnormal immune signaling plays an important role in the pathophysiology of depression. ${ }^{64}$ The levels of factors associated with immune function, like pro-inflammatory factors, acute phase proteins, cellular adhesion molecules and chemokines, have been found to be higher in depressed patients than in others, ${ }^{65}$ and these chemokines can elicit behavior change in animals that are homologous to depression. Moreover, it has been shown that pro-inflammatory factors interact with the pathophysiological domains of depression, including synaptic plasticity. ${ }^{66}$ Abnormal immune signaling of the brain leads to elevated levels of factors associated with immune function and stimulates the activity of serotonin (5-HT) and dopamine (DA) neurons, thereby accelerating the reuptake of central monoamine neurotransmitters and reducing the synaptic neurotransmitter concentration, and influencing the synaptic microenvironment. ${ }^{67}$

Oxidative stress has historically been considered to be mainly related to neurodegenerative disorders, such as Alzheimer's disease, Huntington's disease and Parkinson's disease. But now, its involvement in neuropsychiatric diseases, including anxiety and depression, is beginning to be recognized. ${ }^{68}$ As a consequence of the biological imbalance between reactive oxygen species (ROS) and antioxidants, oxidative stress can lead to the alteration of biomolecules and the loss of control of the intracellular redox-related signaling pathways. The failure of cells to adapt to the changes in redox homeostasis and the subsequent cell death, together with the damage caused by inflammatory mediators, has been considered as major causes of neuroprogression and hence MDD. ${ }^{69}$ Metaanalysis also suggests that oxidative stress is increased in depression. ${ }^{70}$ Another study predicates that oxidative stress is a major regulator of synaptic function and growth by the activation of Jun-N-terminal kinase (JNK) and its transcriptional effector AP-1 and autophagy. ${ }^{71}$ Overall, the relationship between oxidative stress and the synaptic microenvironment deserves more attention.

Mitochondria are synthesized in nerve cells, transported along axons to nerve endings and aggregated and provide energy for the transmission and release of neurotransmitters in synapses through oxidative phosphorylation. ${ }^{72}$ The reduction or dysfunction of mitochondria inside synapses resulting from various causes will change the state of energy supply in the local microenvironment of the synapse and affect the synapse function. One study using a super-resolution microscope to observe the mitochondria in the synapses of neurons revealed that dendritic mitochondria exist as stable compartments of single or multiple filaments. If these local mitochondrial compartments are depleted, both the plasticity and the stimulus-induced synaptic translation are abolished. ${ }^{73}$ The mitochondrial ROS act as synaptic activity sentinels that can regulate the phenotypical consequences of forced synaptic inactivity. ${ }^{74}$ Meanwhile, mitochondrial dysfunction can also be used as a trigger, leading to insufficient adenosine triphosphate production and a lack of energy in the external environment of the synapse. Such balance disruption will lead to the formation of multiple pathological products in the microenvironment and cause synaptic dysfunction. ${ }^{75}$ One study using the isobaric tag for relative and absolute quantitation (iTRAQ)-based quantitative proteomics reported results that suggest synaptic mitochondrial dysfunction in the hippocampus of rats susceptible to chronic mild stress. ${ }^{76}$ Results from another study imply that mitochondria play a critical role in synaptic plasticity accompanied by increased BDNF levels, with a significant positive correlation between BDNF levels and mitochondria and synapse numbers. ${ }^{77}$

In addition, neurotransmitter transmission is also affected by the synaptic microenvironment. It has been confirmed that under different conditions the release ability and movement mode of synaptic vesicles containing neurotransmitters are greatly different. ${ }^{78}$ A review summarized the three ways of neurotransmitter release - synchronous, asynchronous and spontaneous - and analyzed the similarities and differences in the mechanisms of these three release methods. The results show that the modes of these three ways of release have key fusion processes in common but may differ in the source of and necessity for 
$\mathrm{Ca}(2+)$ to trigger release and in the identity of the $\mathrm{Ca}(2+)$ sensor for release. ${ }^{79}$ However, mitochondria always act as sensors and regulators of calcium signaling, ${ }^{80}$ therefore, the release of neurotransmitters is affected by mitochondria. In addition, neurotransmitter release from axon terminals is regulated by glial cells. One study found that glutamate release in the brain is impaired in mice lacking low-density lipoprotein receptor-related protein 4 (Lrp4), a protein that is critical for neuromuscular junction formation, and revealed compromised release probability in astrocyte-specific Lrp4 knockout mice. ${ }^{81}$ Another study suggests that chronic mild stress impairs GABA release and uptake by upregulating miRNAs and downregulating mRNAs and proteins, which may constitute the subcellular and molecular mechanisms for the lowered GABA tone in major depression. ${ }^{82}$ All these findings indicate that dynamic changes in synaptic microenvironment will result in abnormal intracellular transport of neurons and synaptic vesicle transport, which is related to depression.

\section{Clinical Significance of Studying the Synaptic Microenvironment in Depression}

\section{Synaptic Microenvironment and Neural Plasticity}

Neuroplasticity is one of the most important characteristics of the central nervous system (CNS) and encompasses the vast changes occurring in all of the constituents of the CNS throughout the life of an individual in response to stimuli. ${ }^{83}$ Moreover, neuroplasticity is manifested as changes in brain memory and learning, body motor function and mental activity on the macro level, while on the micro level it refers to structural and functional alterations in neuronal synapses. ${ }^{84}$ Various stimuli cause changes in plasticity of neurons, resulting in synaptic degeneration and loss, and leading to the remodeling of new synapses and the changes of the transmission efficiency between synapses; this can affect the occurrence and development of neurological diseases. ${ }^{85}$

However, synaptic plasticity is also an important aspect of neuroplasticity and is affected by the synaptic microenvironment. The synaptic microenvironment can be used as one of the targets of neuronal plasticity to improve the synaptic microenvironment, speed up the synaptic transmission efficiency, help the establishment of damaged neuron information and promote damage repair.

\section{Biomarkers of the Synaptic \\ Microenvironment}

Biomarkers for depression are still controversial. Evidence from clinical research and animal models has long suggested that mechanisms and biomarkers associated with synaptic plasticity have always focused on Alzheimer's disease and cognitive decline. ${ }^{86,87}$ As an important member of the neurotrophic family of secreted proteins, BDNF and its receptor participate in a variety of CNS functions, like neuronal growth and differentiation, synaptic plasticity $^{88}$ and its role in depression. Therefore, BDNF has become one of the biomarkers of synaptic plasticity. ${ }^{89}$

Regrettably, as the internal and external environment of synapses, the biological markers of the synaptic microenvironment have not yet been determined. Strengthening the research of biological indicators of the synaptic microenvironment for depression can provide references for rapid clinical diagnosis and treatment.

\section{A Breakthrough in Molecular Imaging in Depression}

Molecular imaging techniques such as magnetic resonance (MR) spectroscopy and positron emission computed tomography (PET) have been used to explore the molecular pathophysiology of depression and evaluate treatment response. $^{90}$

However, due to the complexity and heterogeneity of depression, there are still major challenges for MR spectroscopy and PET, which are limited to examining a few metabolites or a single radioligand at a time. ${ }^{91}$ In this case, the synaptic microenvironment can be used as a breakthrough in molecular imaging of depression. It can monitor the interaction between the synaptic microenvironment and its surroundings in real time, and analyze the relationship between them from multiple levels, so as to promote the dynamic visualization development of biological research.

\section{Synaptic Microenvironment as Potential Drug Target in Antidepressant Treatment} Taking the synaptic microenvironment as the intervention target can provide new ideas for antidepressant treatment. As mentioned above, the synaptic microenvironment of depression is affected by abnormal immune signaling, oxidative stress, neurotransmitters, mitochondrial energy metabolism and other factors. However, most of the current research on depression disease focuses on a single 
influencing factor, ${ }^{92}$ while ignoring the interaction between various factors. The study of the synaptic microenvironment can open up a new window for solving this problem, and the comprehensive research of factors affecting synaptic structure and function can provide a target for establishing the overall level of synaptic.

\section{Conclusion and Outlook}

This is an exciting time for the study of the synaptic microenvironment and synaptic plasticity in depression. The changes induced by neuroplasticity in depression have been described in detail at different levels of the CNS, and all these aspects are strictly intertwined. Indeed, emerging study supports the view that even a small structural modification of one of the neuronal networks may cause alterations in synaptic function and affect the synaptic microenvironment of neurons. At the same time, in the pathological process of depression, the synaptic microenvironment is also affected by abnormal immune signaling, excessive oxidative stress, mitochondrial dysfunction and neurotransmitter deficiency. Unfortunately, the synaptic microenvironment has not yet attracted the attention of depression scholars, but it may have more clinical value.

The importance of the synaptic microenvironment for the maintenance of synaptic homeostasis and synaptic plasticity suggests that it may play a key role in the pathogenesis of depression. Further research on the role of the synaptic microenvironment and synaptic plasticity will shed new light onto potential mechanisms contributing to depressive disorders and provide targets for antidepressant treatment.

\section{Disclosure}

Rongjuan Guo reports grants from Beijing Natural Science Foundation of China (Grant No.7202120), during the conduct of the study. The authors report no other conflicts of interest in this work.

\section{References}

1. Muñoz RF, Bunge EL. Prevention of depression worldwide: a wake-up call. Lancet Psychiatry. 2016;3:306-307. doi:10.1016/S2215-0366(15) 00555-6

2. Stubbs B, Vancampfort D, Veronese N, et al. Depression and physical health multimorbidity: primary data and country-wide meta-analysis of population data from 190593 people across 43 low- and middle-income countries. Psychol Med. 2017;47:2107-2117. doi:10.1017/S0033291717000551

3. Smith K. Mental health: a world of depression. Nature. 2014;515:181. doi: $10.1038 / 515180 \mathrm{a}$
4. GBD 2017 Disease and Injury Incidence and Prevalence Collaborators. Global, regional, and national incidence, prevalence, and years lived with disability for 354 diseases and injuries for 195 countries and territories, 1990-2017: a systematic analysis for the Global Burden of Disease Study 2017. Lancet. 2018;392:1789-1858. doi:10.1016/S0140-6736(18)32279-7

5. Ren X, Yu S, Dong W, Yin P, Xu X, Zhou M. Burden of depression in China, 1990-2017: findings from the global burden of disease study 2017. J Affect Disord. 2020;268:95-101. doi:10.1016/j.jad.2020.03. 011

6. Herrman H, Kieling C, McGorry P, Horton R, Sargent J, Patel V. Reducing the global burden of depression: a lancet-world psychiatric association commission. Lancet. 2019;393:e42-e43. doi:10.1016/ S0140-6736(18)32408-5

7. De Pittà M, Brunel N, Astrocytes VA. Orchestrating synaptic plasticity? Neuroscience. 2016;323:43-61. doi:10.1016/j. neuroscience.2015.04.001

8. McLeod F, Salinas PC. Wnt proteins as modulators of synaptic plasticity. Curr Opin Neurobiol. 2018;53:90-95. doi:10.1016/j. conb.2018.06.003

9. Marsden WN. Synaptic plasticity in depression: molecular, cellular and functional correlates. Prog Neuropsychopharmacol Biol Psychiatry. 2013;43:168-184. doi:10.1016/j.pnpbp.2012.12.012

10. Singh A, Abraham WC. Astrocytes and synaptic plasticity in health and disease. Exp Brain Res. 2017;235:1645-1655. doi:10.1007/ s00221-017-4928-1

11. Yates D. Synaptic plasticity: microglial cell-mediated depression. Nat Rev Neurosci. 2014;15:280. doi:10.1038/nrn3733

12. Levin SG, Godukhin OV. Modulating effect of cytokines on mechanisms of synaptic plasticity in the brain. Biochemistry (Mosc). 2017;82:264-274. doi:10.1134/S000629791703004X

13. Bijata M, Labus J, Guseva D, et al. Synaptic remodeling depends on signaling between serotonin receptors and the extracellular matrix. Cell Rep. 2017;19:1767-1782. doi:10.1016/j.celrep.2017.05.023

14. Harris KP, Littleton JT. Transmission, development, and plasticity of synapses. Genetics. 2015;201:345-375. doi:10.1534/genetics.115.176529

15. Südhof TC. Towards an understanding of synapse formation. Neuron. 2018;100:276-293. doi:10.1016/j.neuron.2018.09.040

16. Ménard C, Hodes GE, Russo SJ. Pathogenesis of depression: insights from human and rodent studies. Neuroscience. 2016;321:138-162. doi:10.1016/j.neuroscience.2015.05.053

17. Malhi GS, Mann JJ. Depression. Lancet. 2018;392:2299-2312. doi:10.1016/S0140-6736(18)31948-2

18. Duman RS, Aghajanian GK, Sanacora G, Krystal JH. Synaptic plasticity and depression: new insights from stress and rapid-acting antidepressants. Nat Med. 2016;22:238-249. doi:10.1038/nm.4050

19. Seo JS, Wei J, Qin L, Kim Y, Yan Z, Greengard P. Cellular and molecular basis for stress-induced depression. Mol Psychiatry. 2017;22(10):1440-1447. doi:10.1038/mp.2016.118.

20. Fried EI, Nesse RM, Guille C, Sen S. The differential influence of life stress on individual symptoms of depression. Acta Psychiatr Scand. 2015;131(6):465-471. doi:10.1111/acps.12395.

21. Hao Y, Ge H, Sun M, Gao Y. Selecting an appropriate animal model of depression. Int J Mol Sci. 2019;20(19):4827. doi:10.3390/ ijms20194827.

22. Czéh B, Fuchs E, Wiborg O, Simon M. Animal models of major depression and their clinical implications. Prog Neuropsychopharmacol Biol Psychiatry. 2016;64:293-310. doi:10.1016/j.pnpbp.2015.04.004.

23. Wang C, Guo J, Guo R. Effect of XingPiJieYu decoction on spatial learning and memory and cAMP-PKA-CREB-BDNF pathway in rat model of depression through chronic unpredictable stress. $B M C$ Complement Altern Med. 2017;17:73. doi:10.1186/s12906-0161543-9

24. Sterley TL, Baimoukhametova D, Füzesi T, et al. Social transmission and buffering of synaptic changes after stress. Nat Neurosci. 2018;21 (3):393-403. doi:10.1038/s41593-017-0044-6 
25. Licznerski P, Duman RS. Remodeling of axo-spinous synapses in the pathophysiology and treatment of depression. Neuroscience. 2013;251:33-50. doi:10.1016/j.neuroscience.2012.09.057

26. Lesuis SL, Lucassen PJ, Krugers HJ. Early life stress impairs fear memory and synaptic plasticity; a potential role for GluN2B. Neuropharmacology. 2019;149:195-203. doi:10.1016/j. neuropharm.2019.01.010.

27. Belleau EL, Treadway MT, Pizzagalli DA. The impact of stress and major depressive disorder on hippocampal and medial prefrontal cortex morphology. Biol Psychiatry. 2019;85:443-453. doi:10.1016/ j.biopsych.2018.09.031

28. Fabbri C, Crisafulli C, Calati R, et al. Neuroplasticity and second messenger pathways in antidepressant efficacy: pharmacogenetic results from a prospective trial investigating treatment resistance. Eur Arch Psychiatry Clin Neurosci. 2017;267(8):723-735. doi:10.1007/s00406-017-0766-1.

29. Bansal R, Hellerstein DJ, Peterson BS. Evidence for neuroplastic compensation in the cerebral cortex of persons with depressive illness. Mol Psychiatry. 2018;23(2):375-383. doi:10.1038/mp.2017.34.

30. Popoli M, Gennarelli M, Racagni G. Modulation of synaptic plasticity by stress and antidepressants. Bipolar Disord. 2002;4:166-182. doi:10.1034/j.1399-5618.2002.01159.x

31. Zanos P, Gould TD. Mechanisms of ketamine action as an antidepressant. Mol Psychiatry. 2018;23:801-811. doi:10.1038/ mp.2017.255

32. Krzystyniak A, Baczynska E, Magnowska M, et al. Prophylactic ketamine treatment promotes resilience to chronic stress and accelerates recovery: correlation with changes in synaptic plasticity in the CA3 subregion of the hippocampus. Int J Mol Sci. 2019;20:1726. doi:10.3390/ijms20071726

33. Moda-Sava RN, Murdock MH, Parekh PK, et al. Sustained rescue of prefrontal circuit dysfunction by antidepressant-induced spine formation. Science. 2019;364(6436):eaat8078. doi:10.1126/science. aat8078.

34. Yao N, Skiteva O, Zhang X, Svenningsson P, Chergui K. Ketamine and its metabolite (2R,6R)-hydroxynorketamine induce lasting alterations in glutamatergic synaptic plasticity in the mesolimbic circuit. Mol Psychiatry. 2018;23(10):2066-2077. doi:10.1038/mp.2017.239.

35. Eid RS, Gobinath AR, Galea LAM. Sex differences in depression: insights from clinical and preclinical studies. Prog Neurobiol. 2019;176:86-102. doi:10.1016/j.pneurobio.

36. Altemus M, Sarvaiya N, Neill Epperson C. Sex differences in anxiety and depression clinical perspectives. Front Neuroendocrinol. 2014;35 (3):320-330. doi:10.1016/j.yfrne.2014.05.004.

37. Tozzi A, Durante V, Manca P, et al. Bidirectional synaptic plasticity is driven by sex neurosteroids targeting estrogen and androgen receptors in hippocampal CA1 pyramidal neurons. Front Cell Neurosci. 2019;13:534. doi:10.3389/fncel.2019.00534.

38. Liu RH, Xue M, Li XH, Zhuo M. Sex difference in synaptic plasticity in the anterior cingulate cortex of adult mice. Mol Brain. 2020;13 (1):41. doi:10.1186/s13041-020-00583-8.

39. Ma L, Xu Y, Wang G, Li R. What do we know about sex differences in depression: A review of animal models and potential mechanisms. Prog Neuropsychopharmacol Biol Psychiatry. 2019;89:48-56. doi:10.1016/j.pnpbp.2018.08.026

40. Hodes GE, Walker DM, Labonté B, Nestler EJ, Russo SJ. Understanding the epigenetic basis of sex differences in depression. J Neurosci Res. 2017;95(1-2):692-702. doi:10.1002/jnr.23876.

41. van Loo HM, Aggen SH, Gardner CO, Kendler KS. Sex similarities and differences in risk factors for recurrence of major depression. Psychol Med. 2018;48(10):1685-1693. doi:10.1017/S003329171700 3178 .

42. Song I, Dityatev A. Crosstalk between glia, extracellular matrix and neurons. Brain Res Bull. 2018;136:101-108. doi:10.1016/j. brainresbull.2017.03.003
43. Dityatev A, Schachner M. Extracellular matrix molecules and synaptic plasticity. Nat Rev Neurosci. 2003;4:456-468. doi:10.1038/nrn1115

44. Roll L, Faissner A. Tenascins in CNS lesions. Semin Cell Dev Biol. 2019;89:118-124. doi:10.1016/j.semcdb.2018.09.012

45. Rhodes KE, Fawcett JW. Chondroitin sulphate proteoglycans: preventing plasticity or protecting the CNS? J Anat. 2004;204:33-48. doi:10.1111/j.1469-7580.2004.00261.x

46. Stranahan AM, Erion JR, Wosiski-Kuhn M. Reelin signaling in development, maintenance, and plasticity of neural networks. Ageing Res Rev. 2013;12:815-822. doi:10.1016/j.arr.2013.01.005

47. Park YK, Goda Y. Integrins in synapse regulation. Nat Rev Neurosci. 2016;17:745-756. doi:10.1038/nrn.2016.138

48. Šekeljić V, Andjus PR. Tenascin-C and its functions in neuronal plasticity. Int J Biochem Cell Biol. 2012;44:825-829. doi:10.1016/j. biocel.2012.02.014

49. Dityatev A, Schachner M, Sonderegger P. The dual role of the extracellular matrix in synaptic plasticity and homeostasis. Nat Rev Neurosci. 2010;11:735-746. doi:10.1038/nrn2898

50. Bijata M, Labus J, Guseva D, et al. Synaptic remodeling depends on signaling between serotonin receptors and the extracellular matrix. Cell Rep. 2017;19(9):1767-1782. doi:10.1016/j.celrep.2017.05.023.

51. Muoio V, Persson PB, Sendeski MM. The neurovascular unit - concept review. Acta Physiol (Oxf). 2014;210:790-798. doi:10.1111/apha.12250

52. De Luca C, Colangelo AM, Virtuoso A, Alberghina L, Papa M. Neurons, glia, extracellular matrix and neurovascular unit: a systems biology approach to the complexity of synaptic plasticity in health and disease. Int J Mol Sci. 2020;21:1539. doi:10.3390/ ijms21041539

53. Ardalan M, Wegener G, Polsinelli B, Madsen TM, Nyengaard JR. Neurovascular plasticity of the hippocampus one week after a single dose of ketamine in genetic rat model of depression. Hippocampus. 2016;26(11):1414-1423. doi:10.1002/hipo.22617.

54. De Pittà M, Volman V, Berry H, Ben-Jacob E. A tale of two stories: astrocyte regulation of synaptic depression and facilitation. PLoS Comput Biol. 2011;7:e1002293. doi:10.1371/journal.pcbi.1002293

55. Rial D, Lemos C, Pinheiro H, et al. Depression as a glial-based synaptic dysfunction. Front Cell Neurosci. 2016;9:521. doi:10.3389/ fncel.2015.00521

56. Blanco-Suarez E, Liu TF, Kopelevich A, Allen NJ. Astrocytesecreted chordin-like 1 drives synapse maturation and limits plasticity by increasing synaptic GluA2 AMPA receptors. Neuron. 2018;100 (5):1116-1132.e13. doi:10.1016/j.neuron.2018.09.043.

57. Adamsky A, Kol A, Kreisel T, et al. Astrocytic activation generates de novo neuronal potentiation and memory enhancement. Cell. 2018;174(1):59-71.e14. doi:10.1016/j.cell.2018.05.002.

58. Nguyen PT, Dorman LC, Pan S, et al. Microglial remodeling of the extracellular matrix promotes synapse plasticity. Cell. 2020;182 (2):388-403.e15. doi:10.1016/j.cell.2020.05.050.

59. Delekate A, Zagrebelsky M, Kramer S, Schwab ME, Korte M. NogoA restricts synaptic plasticity in the adult hippocampus on a fast time scale. Proc Natl Acad Sci USA. 2011;108:2569-2574. doi:10.1073/pnas.1013322108

60. Hughes AN, Appel B. Oligodendrocytes express synaptic proteins that modulate myelin sheath formation. Nat Commun. 2019;10 (1):4125. doi:10.1038/s41467-019-12059-y.

61. Jang M, Gould E, Xu J, Kim EJ, Kim JH. Oligodendrocytes regulate presynaptic properties and neurotransmission through BDNF signaling in the mouse brainstem. Elife. 2019;8:e42156. doi:10.7554/eLife.42156.

62. Dantzer R. Neuroimmune interactions: from the brain to the immune system and vice versa. Physiol Rev. 2018;98:477-504. doi:10.1152/ physrev.00039.2016

63. Dantzer R, O’Connor JC, Freund GG, Johnson RW, Kelley KW. From inflammation to sickness and depression: when the immune system subjugates the brain. Nat Rev Neurosci. 2008;9:46-56. doi:10.1038/nrn2297 
64. Köhler O, Benros ME, Nordentoft $M$, et al. Effect of anti-inflammatory treatment on depression, depressive symptoms, and adverse effects: a systematic review and meta-analysis of randomized clinical trials. JAMA Psychiatry. 2014;71:1381-1391. doi:10.1001/jamapsychiatry.2014.1611

65. Lotrich FE. Inflammatory cytokine-associated depression. Brain Res. 2015;1617:113-125. doi:10.1016/j.brainres.2014.06.032

66. Raison CL, Capuron L, Miller AH. Cytokines sing the blues: inflammation and the pathogenesis of depression. Trends Immunol. 2006;27:24-31. doi:10.1016/j.it.2005.11.006

67. Wang T, Sun X, Qin W, et al. From inflammatory reactions to neurotransmitter changes: implications for understanding the neurobehavioral changes in mice chronically infected with Toxoplasma gondii. Behav Brain Res. 2019;359:737-748. doi:10.1016/j.bbr.20 18.09.011

68. Salim S. Oxidative stress and the central nervous system. J Pharmacol Exp Ther. 2017;360:201-205. doi:10.1124/jpet.116.23 7503

69. Bakunina N, Pariante CM, Zunszain PA. Immune mechanisms linked to depression via oxidative stress and neuroprogression. Immunology. 2015;144(3):365-373. doi:10.1111/imm.12443.

70. Black CN, Bot M, Scheffer PG, Cuijpers P, Penninx BW. Is depression associated with increased oxidative stress? A systematic review and meta-analysis. Psychoneuroendocrinology. 2015;51:164-175. doi:10.1016/j.psyneuen.2014.09.025

71. Milton VJ, Sweeney ST. Oxidative stress in synapse development and function. Dev Neurobiol. 2012;72:100-110. doi:10.1002/dneu.20957

72. Fedorovich SV, Waseem TV, Puchkova LV. Biogenetic and morphofunctional heterogeneity of mitochondria: the case of synaptic mitochondria. Rev Neurosci. 2017;28:363-373. doi:10.1515/ revneuro-2016-0077

73. Rangaraju V, Lauterbach M, Schuman EM. Spatially stable mitochondrial compartments fuel local translation during plasticity. Cell. 2019;176(1-2):73-84.e15. doi:10.1016/j.cell.2018.12.013.

74. Sidlauskaite E, Gibson JW, Megson IL, et al. Mitochondrial ROS cause motor deficits induced by synaptic inactivity: implications for synapse pruning. Redox Biol. 2018;16:344-351. doi:10.1016/j. redox.2018.03.012.

75. Todorova V, Blokland A. Mitochondria and synaptic plasticity in the mature and aging nervous system. Curr Neuropharmacol. 2017;15:166-173. doi:10.2174/1570159x14666160414111821

76. Xie H, Huang H, Tang M, et al. iTRAQ-based quantitative proteomics suggests synaptic mitochondrial dysfunction in the hippocampus of rats susceptible to chronic mild stress. Neurochem Res. 2018;43(12):2372-2383. doi:10.1007/s11064-018-2664-y.

77. Chen F, Danladi J, Ardalan M, et al. Role of mitochondria in BDNF-associated synaptic plasticity after one-week vortioxetine treatment. Int J Neuropsychopharmacol. 2018;21(6):603-615. doi:10. 1093/ijnp/pyy022.
78. Rizo J, Xu J. The synaptic vesicle release machinery. Annu Rev Biophys. 2015;44:339-367. doi:10.1146/annurev-biophys-060414034057

79. Kaeser PS, Regehr WG. Molecular mechanisms for synchronous, asynchronous, and spontaneous neurotransmitter release. Annu Rev Physiol. 2014;76:333-363. doi:10.1146/annurev-physiol-021113170338.

80. Rizzuto R, De Stefani D, Raffaello A, Mammucari C. Mitochondria as sensors and regulators of calcium signalling. Nat Rev Mol Cell Biol. 2012;13(9):566-578. doi:10.1038/nrm3412.

81. Sun XD, Li L, Liu F, et al. Lrp4 in astrocytes modulates glutamatergic transmission. Nat Neurosci. 2016;19(8):1010-1018. doi:10.1038/ nn. 4326 .

82. Ma K, Xu A, Cui S, Sun MR, Xue YC, Wang JH. Impaired GABA synthesis, uptake and release are associated with depression-like behaviors induced by chronic mild stress. Transl Psychiatry. 2016;6 (10):e910. doi:10.1038/tp.2016.181.

83. Cramer SC, Sur M, Dobkin BH, et al. Harnessing neuroplasticity for clinical applications. Brain. 2011;134:1591-1609. doi:10.1093/brain/ awr039

84. Hall FS, Li A, Li B. Neural plasticity in mood disorders. Neural Plast. 2018;2018:3745251. doi:10.1155/2018/3745251

85. Karpova NN, Sales AJ, Joca SR. Epigenetic basis of neuronal and synaptic plasticity. Curr Top Med Chem. 2017;17:771-793. doi:10.2174/1568026616666160414124628

86. Bos I, Vos S, Verhey F, et al. Cerebrospinal fluid biomarkers of neurodegeneration, synaptic integrity, and astroglial activation across the clinical Alzheimer's disease spectrum. Alzheimers Dement. 2019;15:644-654. doi:10.1016/j.jalz.2019.01.004

87. Spinelli M, Fusco S, Grassi C. Brain insulin resistance and hippocampal plasticity: mechanisms and biomarkers of cognitive decline. Front Neurosci. 2019;13:788. doi:10.3389/fnins.2019.00788

88. Sasi M, Vignoli B, Canossa M, Blum R. Neurobiology of local and intercellular BDNF signaling. Pflugers Arch. 2017;469:593-610. doi:10.1007/s00424-017-1964-4

89. Gururajan A, Clarke G, Dinan TG, Cryan JF. Molecular biomarkers of depression. Neurosci Biobehav Rev. 2016;64:101-133. doi:10.1016/j.neubiorev.2016.02.011

90. Lee TS, Quek SY, Krishnan KR. Molecular imaging for depressive disorders. AJNR Am J Neuroradiol. 2014;35:S44-S54. doi:10.3174/ ajnr.A3965

91. Li Z, He Y, Tang J, Zong X, Hu M, Chen X. Molecular imaging of striatal dopamine transporters in major depression-a meta-analysis. J Affect Disord. 2015;174:137-143. doi:10.1016/j.jad.2014.11.045

92. Kern N, Sheldrick AJ, Schmidt FM, Minkwitz J. Neurobiology of depression and novel antidepressant drug targets. Curr Pharm Des. 2012;18:5791-5801. doi:10.2174/138161212803523581
Neuropsychiatric Disease and Treatment

\section{Publish your work in this journal}

Neuropsychiatric Disease and Treatment is an international, peerreviewed journal of clinical therapeutics and pharmacology focusing on concise rapid reporting of clinical or pre-clinical studies on a range of neuropsychiatric and neurological disorders. This journal is indexed on PubMed Central, the 'PsycINFO' database and CAS, and is the official journal of The International Neuropsychiatric Association (INA). The manuscript management system is completely online and includes a very quick and fair peer-review system, which is all easy to use. Visit http://www.dovepress.com/testimonials.php to read real quotes from published authors. 